\title{
Der (National-)Staat und die Europäische Union- die EU auf dem Fusionspfad?
}

von Wolfgang Wessels

Mit dem Vertrag von Lissabon steht die Grundsatzfrage nach der Natur der europäischen Integration und gleichzeitig der Zukunft des (National-)Staates in Europa erneut im Zentrum einer lebhaften wissenschaftlichen und politischen Debatte. Ausgehend von Analysen des Lissabonner Vertragswerks und Argumentationslinien des Verfassungsgerichtsurteils zum Lissabonner Vertrag skizziert der Beitrag einen Satz von Modellen, um Beziehungsmuster zwischen der Europäischen Union und dem gewachsenen Nationalstaat zu identifizieren. Dabei wird der Vertrag von Lissabon als Stärkung und weiterer Ausbau eines „Fusionspfades" gesehen; dieser Prozess ist durch das Interesse der Mitgliedstaaten zu erklären, eine zunehmende Anzahl von vitalen Problemen durch die EU effizient und effektiv zu bewältigen. Zentrale Akteure sind dabei die Staats- und Regierungschefs im Europäischen Rat. ${ }^{1}$

The Lisbon Treaty has fuelled the fundamental debate about the European integration and the future of the European (nation) state. With a variation of analyses of the Lisbon Treaty and major lines of the Lisbon Judgment of the German Constitutional Court, this article identifies a set of models of relations between the European Union and the traditional European nation state. The Lisbon Treaty is seen as a continuation and strengthening of a path characterised by "fusion": this process is to be explained by the search of Member States to solve major vital problems efficiently and effectively within the EU system. Major actors shaping this trend are the heads of state and government in the European Council.

\section{Der (National-)Staat und die Europäische Integration: eine Schlüs- selfrage}

Seit Beginn dessen, was wir bewusst unscharf und mehrdeutig „Europäische Integration“ nennen, steht immer wieder eine Schlüsselfrage im Zentrum von Grundsatzdebatten: In welcher Beziehung steht dieses schwer fassbare politische System im Werden zum traditionellen (National-)Staat und zu dessen Wandel? Empirische Studien untersuchen gegenseitige Einwirkungen und Wechselbezie-

1 Für Zu- und Mitarbeit danke ich Frau Maria Schäfer. 
hungen zwischen mehreren Ebenen staatlichen Handelns; normativ wird - unter Bezugnahme auf Kriterien der Problemlösungsfähigkeit und Legitimität - eine intensive Diskussion über optimale Formen der Gestaltung dieses Systems geführt, das häufig - aufgrund seiner hybriden Natur - als ,sui generis “ bezeichnet wird.

Das Vertragswerk von Lissabon hat zu einer erneuten Renaissance dieser Grundsatzdebatte geführt, die nicht nur den Charakter der Union, sondern vielmehr auch Grundpositionen zur Zukunft des Staates in Europa aufgreift und vertieft. Einen sicherlich wirkungsmächtigen Satz von Antworten auf diese Schlüsselfrage liefert das Urteil des deutschen Bundesverfassungsgerichts ${ }^{2}$ zur Vereinbarkeit des Vertrags von Lissabon mit dem deutschen Grundgesetz. Die Karlsruher Richter haben erneut - explizit und implizit - fundamentale Kontroversen zu Grundfragen der europäische Integration in Bezug zur Staatlichkeit der Mitgliedstaaten herausgearbeitet und für die Zukunft - zumindest deutscher Integrationsstrategien - richtungweisende Vorgaben formuliert, die freilich auch unterschiedlich interpretiert und angewandt werden können. Der lange Schatten dieses Dokuments auf die Gestaltung des EU-Systems ist für viele Experten offensichtlich: „Generationen von Politikern werden das Urteil wieder und wieder lesen müssen. “3

Zur Analyse des Vertragswerks ordnet dieser Beitrag den Vertrag von Lissabon in den historischen Integrationsprozess der vergangenen sechs Jahrzehnte ein. Das Dokument ist als erneuter Versuch der Mitgliedstaaten zu sehen, eine Balance zwischen mehreren Fundamentalzielen herzustellen. Nach dieser Analyse haben die nationalstaatlichen Vertragsschöpfer - bei der jahrzehntelangen Gestaltung des EU-Systems und so auch erneut beim Vertrag von Lissabon - drei Grundaufgaben ${ }^{4}$ verfolgt und dabei versucht, ein „magisches Dreieck“ (vgl. Abbildung 1) neu auszutarieren.

Einerseits zeigen Mitgliedstaaten immer wieder eine starke Neigung, Probleme zwischenstaatlicher und globaler Natur mit Hilfe des EU-Systems anzugehen

2 BVerfG, Urt. v. 30.06. 2009, 2 BvE 2/08 u.a. (Vertrag von Lissabon), abgedruckt in: Neue juristische Wochenschrift, 62/31 (2009), 2267-2295.

3 Tomuschat, C.: The Ruling of the German Constitutional Court on the Treaty of Lisbon, in: German Law Journal, 10/8 (2009), 1259-1262, hier 1259 (Übersetzung d. Verf.).

4 Vgl. zur Ableitung dieser Zielvorgaben im EU-Kontext u.a. Spiegelstrich 7 der Präambel des Vertrags über die Europäische Union (Maastricht); Schlussfolgerungen des Europäischen Rates von Laeken, Anlage 1: Erklärung von Laeken zur Zukunft der Europäischen Union, in: Bulletin der Europäischen Union, 12 (2001). 
(„Problembewältigungsinstinkt“). Dem steht das Beharren auf nationale Gestaltungsautonomie (der „Souveränitätsreflex“) gegenüber. Einen dritten Eckpunkt des magischen Dreiecks bildet das Streben nach demokratischer Absicherung (die Legitimitätssuche), die nicht einfach einem der beiden Eckpunkte zugeordnet werden kann. In der Präambel des Lissabonner Vertrags betonen die hohen Vertragsparteien selbst ,den Wunsch, Demokratie und Effizienz in der Arbeit der Organe weiter [durch Vertragsänderungen] zu stärken“.

Abbildung 1: Das „magische Dreieck“

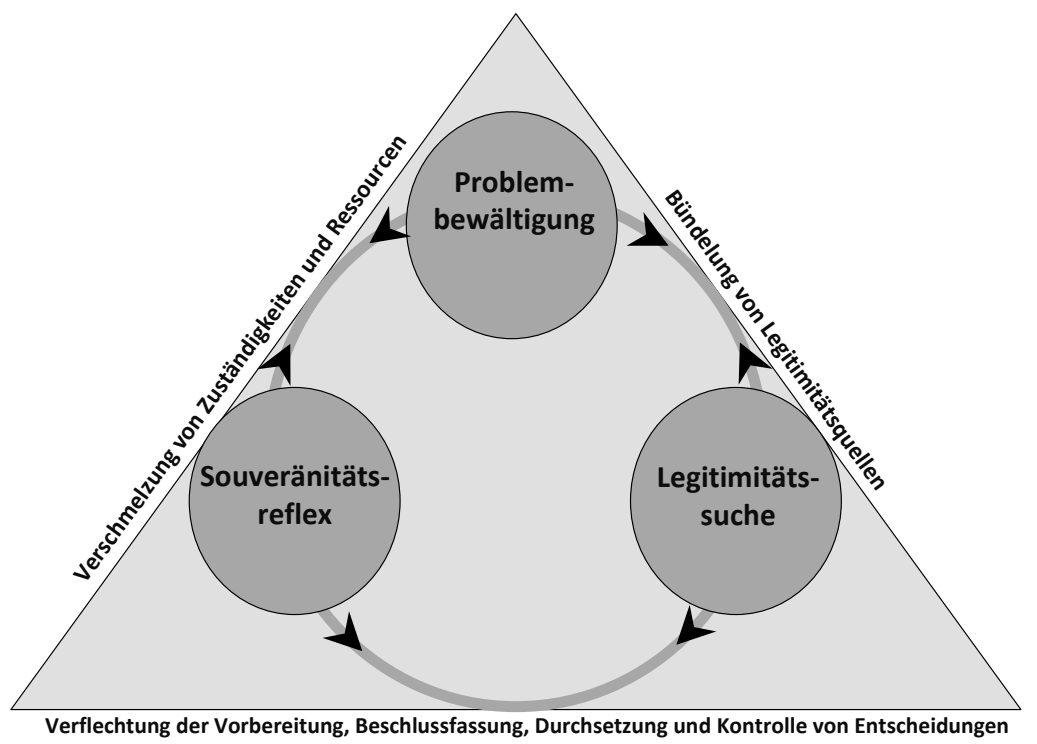

Quelle: Eigene Darstellung.

Die „Hohen Vertragsparteien“ als Vertragsschöpfer haben nicht einen eindeutigen, sondern gleichzeitig mehrere - auch unterschiedliche und widersprüchliche - Wege eingeschlagen, um diese Aufgaben anzugehen. Eine Analyse der Gesamtwirkung des Vertragstextes und noch mehr die Erwartungen bei der konkreten Anwendung in der gelebten Vertragswelt zeigen - so die hier vertretene These - den Ausbau eines Pfades, der durch eine „Fusion“ gekennzeichnet ist. Die „Herren der Verträge ${ }^{\text {“5 }}$ haben danach - teils mit Absicht, teils unbeabsich- 
tigt - einen Prozess gestärkt, bei dem sie vertikal zwischen mehreren Ebenen und horizontal zwischen EU-Organen

- de jure Zuständigkeiten, Ressourcen und Instrumente der Problembewältigung in einer zunehmenden Anzahl von Politikbereichen teilen und de facto gemeinsam nutzen,

- dabei die Beteiligungsmöglichkeiten von Akteuren verschiedener Ebenen in den Verfahren zur Vorbereitung, Beschlussfassung, Durchsetzung und Kontrolle von Entscheidungen verflechten

- und dabei ebenso mehrere und unterschiedliche Legitimationsquellen bündeln.

\section{Antworten aus dem Vertrag von Lissabon: Ein Satz von Modellen}

Um Antworten auf die Ausgangsfrage zu sortieren und um den Lissabonner Vertrag einzuordnen, nutzt dieser Beitrag eine Auswahl prominenter Denkschulen und damit verknüpfte integrationsrelevante Staatsbilder ${ }^{6}$, um einen Satz an Modellen zu skizzieren. Die Eckpunkte dieser Beziehungen sind durch ein Nullsummenspiel zwischen der staatlichen und der EU-Ebene gekennzeichnet: Im Modell der EU als „Bundesstaat“ gewinnt die europäische Ebene Zuständigkeiten und Letztentscheidungsrechte zulasten der nationalen Ebene. Der gewachsene Nationalstaat verliert an Souveränität, welche die mit starken Rechten ausgestatteten Institutionen der europäischen Föderation übernehmen. Im Modell der EU als ,normaler“ internationaler Organisation behält der Staat seine Souveränität; die europäische Ebene bleibt ohne eigenständige Zuständigkeiten und den EU-Institutionen werden keine starken Beteiligungsrechte zugestanden.

Interessant sind dazwischen Mischformen, bei denen ein Positivsummenspiel unterstellt wird. „Offene Staaten“ suchen ihre Problemlösungen durch zwischenstaatliche Kooperation zu verbessern, ohne ihre Souveränität endgültig aufzugeben. Die Charakterisierung der EU als „Staatenverbund“, die das Bundesverfassungsgericht vornimmt, und die Einstufung der EU ,als Rettung des

6 Vgl. u.a. Wessels, W.: Die Öffnung des Staates. Modelle und Wirklichkeit grenzüberschreitender Verwaltungspraxis 1960-1995, Opladen, 2000; Bartolini, S.: Restructuring Europe: Centre Formation, System Building, and Political Structuring Between the Nation State and the European Union, Oxford/New York, 2005; Calliess, C. (Hg.): Verfassungswandel im europäischen Staaten- und Verfassungsverbund, Tübingen, 2007; Deitelhoff, N./Steffek, J.: Was bleibt vom Staat? Demokratie, Recht und Verfassung im globalen Zeitalter, Frankfurt/M., 2009. 
Nationalstaates" signalisieren beide, dass die nationale Souveränität - zumindest de facto - erhalten bzw. sogar durch die EU gestärkt wird.

Bei dem Modell der Fusion werden - als Folge des Problembewältigungsinstinktes - zunehmend Ressourcen de jure und noch mehr de facto geteilt, prozedurale Beteiligungsmöglichkeiten verflochten und Legitimitätsquellen gebündelt.

\section{Die Ablösung des Nationalstaates - der finale Schritt zum Bundesstaat?}

Ein zentraler Ausgangspunkt, auf den auch das Urteil des Bundesverfassungsgerichts deutlich Bezug nimmt, ist der Typ des „Bundesstaates“, der von den Souveränitätsverfechtern häufig mit Merkmalen eines „Superstaates“" ${ }^{\text {"7 }}$ versehen und deshalb abgelehnt wird. Dieses Modell verstehen dagegen Föderalisten als notwendige Weiterentwicklung der Europäischen Union; ${ }^{8}$ aus dieser Sicht stellt sich der gewachsene Nationalstaat nicht nur unzeitgemäß gegen grundsätzliche Tendenzen der Gegenwartsgeschichte, ${ }^{9}$ sondern bildet einen „Irrweg ${ }^{\text {“10 }}$. Eine über die vergangenen Jahrzehnte durchgängige Argumentation betont, dass ,die Nationalstaaten [...] schon zu schwach [sind], um ihre klassischen Funktionen zu erfüllen“111; deshalb wird gefolgert: „Herausforderungen der Globalisierung [kann] nur auf vernünftige Weise [begegnet werden], wenn es gelingt, in der postnationalen Konstellation neue Formen einer demokratischen Selbststeuerung zu entwickeln“'12.

Diese Überlegungen sind nicht erst der Globalisierungsdebatte geschuldet, denn bereits der erste gewählte Bundestag ${ }^{13}$ forderte

„einen Europäischen Bundespakt [...], wie ihn die Präambel und [der damalige, d. Verf.] Artikel 24 des Grundgesetzes für die Bundesrepublik Deutschland vorsieht": Er soll „eine übernationale Bundesgewalt schaffen, die sich auf allgemeine, unmit-

7 Vgl. u.a. Dougan, M.: The Treaty of Lisbon 2007: Winning Minds, Not Hearts, in: Common Market Law Review, 45/3 (2008), 617-703, hier 622.

8 Vgl. Montani, G.: The Bundesverfassungsgericht sentence and the future of the European Union, online unter http://www.federaleurope.org/index.php?id=6719\&tx_ttnews[tt_news] $=2442 \& \mathrm{cHash}=7 \mathrm{cfaf} 7038 \mathrm{a}$.

9 Vgl. Levi, L.: Recent Developments in Federalist Theory, in: The Federalist. A political review, 29/2 (1987), 97-135.

10 Glotz, P.: Der Irrweg des Nationalstaats. Europäische Rede an ein deutsches Publikum, Stuttgart, 1990, $89 \mathrm{ff}$.

11 Ebd., 169.

12 Habermas, J.: Die postnationale Konstellation. Politische Essays, Frankfurt/M., 2006, 134.

13 Das Urteil des Bundesverfassungsgerichts listet in seiner geschichtsbezogenen Erzählung (BVerfG, a. a. O., Rn. 5) diese Resolution des Bundestages nicht auf. 
telbare und freie Wahlen gründet und über gesetzgebende, ausübende und richterliche Kompetenzen verfügt. “14

Das Verfassungsgericht ${ }^{15}$ definiert den Übergang als einen „Identitätswechsel der Bundesrepublik Deutschland“, der „durch Umbildung zu einem Gliedstaat eines europäischen Bundesstaats bewirkt würde“ (Rn. 179). In eindeutiger Übernahme der Befürchtungen einiger Beschwerdeführer zeichnet das Urteil eine „Grenzlinie“ (zum Begriff Rn. 251), die dann überschritten würde, wenn den „Mitgliedstaaten kein ausreichender Raum zur politischen Gestaltung der wirtschaftlichen, kulturellen und sozialen Lebensverhältnisse mehr bleibt“" (Rn. 249). Konkreter führt es eine lange Reihe entsprechender Sachbereiche auf (ebd.). Diese strikte Festlegung von unüberbrückbaren ,Schwellen zum Bundesstaat und zum nationalen Souveränitätsverzicht“ (Rn. 263) wird durch Annahmen zur demokratischen Legitimität, dem Wahlrecht und spezifisch mit dem Verweis auf die Ewigkeitsklausel des Grundgesetzes begründet.

In einem derartig definierten Bundesstaatsmodell würde die EU zu einem Gebilde, das über alle traditionell mit dem ,Staat“" verbundene Charakteristika verfügt: In einem nun größeren Raum basiert das politische System auf „einem Volk“, „einem Territorium“ und einer zentralisierten „Staatsgewalt“ (Rn. 113). Eine zentrale Schwelle ist aus dieser Sicht dann gegeben, wenn sich die EU der „Kompetenz-Kompetenz“ bemächtigte, das heißt, die Union erhielte „Hoheitsrechte [...] aus [deren] Ausübung heraus eigenständig weitere Zuständigkeiten für die Europäische Union begründet werden können“" (Rn. 233).

Diese Schwelle hat der Lissabonner Vertrag aus Sicht des Bundesverfassungsgerichts noch nicht überschritten. Mit Bezug auf die Drei-Elemente-Lehre des Staates argumentiert das Urteil:

- Das „Staatsgebiet [wird nicht aufgegeben]“ (Rn. 344);

- „Die souveräne Staatsgewalt bleibt [...] gewahrt“ (Rn. 299) bzw. „eine gebietsbezogene Staatgewalt besteht [...] unverändert fort" (Rn .344);

- „Die Bundesrepublik wird [...] weiterhin auch über ein Staatsvolk verfügen“; „Die Unionsbürgerschaft [...] konstituiert kein Unionsvolk“ (Rn. 346).

14 Siegler, H.: Europäische politische Einigung, 1949-1968. Dokumentation von Vorschlägen und Stellungnahmen, Bonn u.a., 1968, 1.

15 Alle im Folgenden mit Randnummern in runden Klammern belegten Zitate aus BVerfG, a. a. O. 
Insgesamt erklärt das Urteil: „Die Bundesrepublik Deutschland bleibt [...] ein souveräner Staat“ (Rn. 298), gesichert durch vertragliche „Schutzmechanismen“ (vgl. u. a. Rn. 301 f.). Eine solche Absicherung gilt auch in Zukunft: „Der Fortbestand souveräner Staatsgewalt zeigt sich auch in dem Recht zum Austritt aus der Europäischen Union und wird durch das dem Bundesverfassungsgericht zustehende Letztentscheidungsrecht geschützt“ (Rn. 299).

\section{Die Absicherung des geschlossenen Nationalstaates - die EU nur eine internationale Organisation?}

Am anderen Ende einer möglichen Skala der Beziehungen zwischen Staat und EU steht dem Bundesstaatsmodell das Modell eines ,in sich geschlossenen, in völliger Selbständigkeit handelnden Staates “' ${ }^{\text {16 }}$ gegenüber.

Aufgrund der bereits skizzierten traditionellen staatsrechtlichen Drei-ElementeLehre wird der Staat mit Merkmalen wie ,formaler Autonomie, Monopol der Zwangsanwendung, Abgrenzung von nicht-gouvernementalen Organisationen, Zentralisierung und interne Koordination “17 beschrieben. Daraus folgt, dass „Internationale Organisationen [...] keine eigenständigen Akteure, sondern Vehikel oder Instrument der Staaten “18 sind. Erwartungen an zwischenstaatlich angesiedelte Institutionen wecken deshalb nur „falsche Hoffnungen“19.

Aus diesem Bild ist eine Charakterisierung abzuleiten, die das EU-System nur als eine - vielleicht spezifische - Form einer internationalen Organisation ${ }^{20}$ ausschildert, ihr also keine Kompetenzen und Rechte zuschreibt, welche in die harte Schale des nationalen Verfassungsstaates hineinreichen. Die EU-Organe sollen als agents den mitgliedstaatlichen principals ${ }^{21}$ bei einigen grenzüberschreitenden Problemen helfen bzw. dienen, aber den Kern der Staatlichkeit nicht berühren.

16 Scheuner, U.: Die internationalen Probleme der Gegenwart und die nationale Entscheidungsstruktur, in: Hennis, W./Kielmansegg, P. Graf/Matz, U. (Hg.): Regierbarkeit. Studien zu ihrer Problematisierung, Bd. 1, Stuttgart, 1977, 255-294, hier 290.

17 Tilly, C.: Reflections on the History of European State-Making, in: ders. (Hg.): The Formation of National States in Western Europe, Princeton, 1975, 3-84, hier 34 (Übers. d. Verf.); vgl. auch Poggi, G.: The State: Its Nature, Development, and Prospects, Stanford, 1990, $19 \mathrm{ff}$.

18 Link, W.: Die Neuordnung der Weltpolitik. Grundprobleme globaler Politik an der Schwelle zum 21. Jahrhundert, 3. Aufl., München, 2001, $106 \mathrm{f}$.

19 Vgl. Mearsheimer, J.J.: The False Promise of International Institutions, in: International Security, 19/3 (1994/1995), 5-49.

20 Vgl. Rittberger, V.: Internationale Organisationen. Politik und Geschichte, Opladen, 1995.

21 Vgl. zu diesem Begriffspaar Menon, A./Kassim, H.: The Principal-Agent Approach and the Study of the European Union: Promise Unfulfilled?, in: Journal of European Public Policy, 10/1 (2003), 121-139; 
Die Vielzahl der beobachtbaren Aktivitäten der EU betreffen aus dieser Sicht nur Bereiche der ,, low politics “22. Die Entwicklungen des EU-Systems - einschließlich des Vertrags von Lissabon - sind danach nur als Fußnote in der langen Geschichte der Entwicklung des europäischen Staates zu sehen. Rückblickend kann die Integrationskonstruktion als ein vielleicht a-typisches und relativ überflüssiges Experiment staatlichen Handelns verstanden werden. Der Lissabonner Vertrag ist dann in der Tat das Ende einer Epoche, aber nicht des Staates, sondern der Evolution des EU-Systems. Der status quo des Vertrags ist ein „European constitutional settlement ${ }^{\text {،23 }}$, das den Mitgliedstaaten genügt. Durch Aufbau und Nutzung der EU-Architektur kann der Staat abgesichert werden, ohne jedoch seine eigentliche Natur als in sich geschlossenes System aufzugeben.

In einem gleichgerichteten Verständnis charakterisiert das Urteil des Bundesverfassungsgerichts das Verhältnis der Beziehung der (Mitglied-)Staaten gegenüber der EU eindeutig mit dem Begriff „Herren der Verträge“, das nahe an dem Begriffspaar ,principal " und ,agent “ liegt. Ein derartiges Beziehungsmuster wird auch im Hinweis auf das Austrittsrecht (siehe oben) sowie bei einer Formulierung bezüglich der Beziehungen zwischen beiden Ebenen deutlich: „,die Europäische Union trägt sekundäre, dass heißt delegierte Verantwortung für die ihr übertragenen Aufgaben“ (Rn. 301).

\section{Stärkung und „Rettung“ des offenen Staates?}

Angesichts vielfältiger Ausprägungen zwischenstaatlicher Interaktionen und Formen der Zusammenarbeit haben die Modelle eines „offenen Staates ${ }^{\text {‘2 } 24}$ bzw. der „Öffnung des Staates“'25 an Bedeutung gewonnen, in denen die Mitgliedstaaten eine zunehmende Anzahl von Bereichen öffentlicher Politik gemeinsam

Pollack, M.A.: The Engines of European Integration - Delegations, Agency and Agenda Setting in the EU, Oxford, 2003; Tallberg, J.: The Anatomy of Autonomy: An Institutional Account of Variation in Supranational Influence, in: Journal of Common Market Studies, 38/5 (2000), 843-864.

22 Hoffmann, S.: Obstinate or Obsolete? The Fate of the Nation-State and the Case of Western Europe, in: Daedalus, 95/3 (1966), 862-915, hier 882.

23 Moravcsik, A.: What Can we Learn from the Collapse of the European Constitutional Project?, in: Politische Vierteljahresschrift, 47/2 (2006), 219-241, hier 219.

24 Vgl. zum Begriff insbesondere Vogel, K.: Die Verfassungsentscheidung des Grundgesetzes für eine internationale Zusammenarbeit, Tübingen, 1964, 28, 33; vgl. u. a. auch Klein, E.: Leitsätze. Der Verfassungsstaat als Glied einer Europäischen Gemeinschaft, in: Europarecht, 25/4 (1990), 389-391, hier 389.

25 Vgl. Hobe, S.: Der offene Verfassungsstaat zwischen Souveränität und Interdependenz, Berlin, 1998; Wessels, $W$.: Die Öffnung des Staates, a.a.O. 
regeln. Der offene Staat ordnet sich in „eine pluralistische Gemeinschaft“ ein, an deren Problemverarbeitung er ,teilnimmt“ ${ }^{\star 26}$.

Die Mitgliedstaaten geben zwar nationalstaatliche Autonomie auf, gewinnen aber dafür an zwischenstaatlichen Gestaltungsmöglichkeiten, welche die Wirksamkeit staatlicher Steuerungsinstrumente aus der Sicht der Akteure wesentlich verbessern.

Im Unterschied zum Bundesstaatsmodell gibt der Staat seine Rechte nicht auf, sondern wird zum aktiven Mitspieler. „Der Staat ,verzichtet ${ }^{\star}$ hier nicht, sondern er gibt seiner Tätigkeit lediglich eine andere Richtung. ${ }^{627}$ Der (west-)europäische Nationalstaat wurde durch neue Formen zwischenstaatlicher Problembewältigung de facto gestärkt. ${ }^{28}$

Eine weitere, besondere Ausprägung dieses Modells bildet die These, das EU-

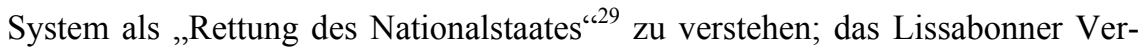
tragswerk dokumentiert danach die gefühlte Notwendigkeit der EU-Mitgliedstaaten, gemeinsam zentrale Themen öffentlicher Politik anzugehen. Die Relevanz europäischer Politikgestaltung ist demnach weitaus größer, als die Bewertung einer funktionellen Ergänzung staatlicher Aufgabenwahrnehmung nach dem Modell der internationale Organisation zunächst erkennen lässt. Aus dieser Sicht ist eine Mitgliedschaft von „vitalem nationalen Interesse ${ }^{\text {‘30 }}$. Danach haben die Mitgliedstaaten im Lissabonner Vertragswerk erneut ihre Fähigkeit festgeschrieben bzw. ausgebaut, anstehende und zukünftige Probleme durch EU-Institutionen zu bewältigen.

Das Verfassungsgericht greift einige Annahmen und Argumente dieser Denkschule auf:

„Die deutsche Verfassung ist auf Öffnung der staatlichen Herrschaftsordnung für das friedliche Zusammenwirken der Nationen und die europäische Integration gerichtet $[\ldots]$ Es handelt sich [...] um freiwillige, gegenseitige und gleichberechtigte Bindung,

26 Vogel, K.: Die Verfassungsentscheidung des Grundgesetzes, a. a. O., 28.

27 Ebd., 31.

$28 \mathrm{Vgl}$. Hoffmann, S.: Reflections on the Nation State in Western Europe Today, in: Journal of Common Market Studies, 21/1-2 (1982), 21-37; Milward, A.: The European Rescue of the Nation State, Berkeley, 1992; Moravcsik, A.: Preferences and Power in the European Community. A Liberal Intergovernmental Approach, in: Journal of Common Market Studies, 31/4 (1993), 437-524.

29 Vgl. Milward, A.: The European Rescue of the Nation-State, a. a. O.

30 Vgl. u.a. Hrbek, R./Wessels, W. (Hg.): EG-Mitgliedschaft: ein vitales Interesse der Bundesrepublik Deutschland?, Bonn, 1984; Diedrichs, U./Wessels, W.: Die neue Europäische Union: im vitalen Interesse Deutschlands?, Berlin, 2006. 
die den Frieden sichert und die politischen Gestaltungsmöglichkeiten durch gemeinsames koordiniertes Handeln stärkt“" (Rn. 220).

Das Urteil unterstreicht die Sinnhaftigkeit der Kooperation: „Nur wer sich aus Einsicht in die Notwendigkeit friedlichen Interessenausgleichs und in die Möglichkeiten gemeinsamer Gestaltung bindet, gewinnt das erforderliche Maß an Handlungsmöglichkeiten, um die Bedingungen einer freien Gesellschaft auch künftig verantwortlich gestalten zu können“" (Rn. 221). Noch deutlicher analysieren die Richter: „Föderalisierung nach innen und Supranationalisierung nach außen können neue bürgerschaftliche Mitwirkungsmöglichkeiten eröffnen [...] Föderale oder supranationale Verflechtungen schaffen Handlungsmöglichkeiten, die sonst auf praktische oder territoriale Grenzen stießen“" (Rn. 247).

Aber das Urteil macht auch das Spannungsverhältnis zwischen Problembewältigungsinstinkt und Legitimitätssuche in einer trade-off-Beziehung deutlich: Diese Handlungsmöglichkeiten

„erschweren aber zugleich die Bildung eines durchsetzungsfähigen Mehrheitswillens, der unmittelbar auf das Volk zurückgeht (Art. 20 Abs. 2 Satz 1 GG). Die Zuordnung von Entscheidungen zu bestimmten verantwortlich Handelnden verliert an Transparenz mit der Folge, dass die Bürger sich bei ihrem Votum kaum an greifbaren Verantwortungszusammenhängen orientieren können“ (Rn. 247).

Aus dieser Analyse zieht das Urteil folgende strategische Schlussfolgerung: „Das Demokratieprinzip setzt deshalb der Übertragung von Hoheitsrechten inhaltliche Grenzen“ (Rn. 247). Und nochmals noch deutlicher formuliert es: Bei ,der Beteiligung Deutschlands an der Europäischen Union [handelt es sich] indes nicht um die Übertragung eines Bundesstaatsmodells auf die europäische Ebene, sondern um die Erweiterung des verfassungsrechtlichen Föderalmodells um eine überstaatlich kooperative Dimension“ (Rn. 277, Hervorhebung d. Verf.). In diesem Sinne betont das Urteil auch mehrfach den „Grundsatz der Europarechtsfreundlichkeit“" (Rn. 225).

\section{Endgültige Bestätigung des Verbundes souveräner Nationalstaaten?}

In der Skala der Modelle ist nicht zuletzt die Kategorie „Staatenverbund“ zu beleuchten, mit dem die Richter die staatsrechtliche Kategorienbildung zum EUSystem nachhaltig prägen wollen. Die Definition des Schlüsselbegriffs lautet:

„Das Grundgesetz ermächtigt mit Art. 23 GG zur Beteiligung und Entwicklung einer als Staatenverbund konzipierten Europäischen Union. Der Begriff des Verbundes erfasst eine enge, auf Dauer angelegte Verbindung souverän bleibender Staaten, die auf 
vertragliche Grundlage öffentliche Gewalt ausübt, deren Grundordnung jedoch allein der Verfügung der Mitgliedstaaten unterliegt und in der die Völker - das heißt die staatsangehörigen Bürger - der Mitgliedstaaten die Subjekte demokratischer Legitimation bleiben“" (Leitsatz 1).

Die vom Gericht selbst erstellte englische Fassung, die ja für den notwendigen europäischen Diskurs ausschlaggebend sein wird, übersetzt und interpretiert den Begriff „Staatenverbund“ als ,, association of national sovereign states “; mit der im entsprechenden deutschen Abschnitt nicht auftauchenden Formulierung ,national“" wird ein verstärkendes Signal ausgestrahlt.

Erneut - wie im Maastricht-Urteil - definiert das Verfassungsgericht das Verhältnis von Mitgliedstaat und Union deutlich:

„Die Ermächtigung zur Übertragung von Hoheitsrechten auf die Europäische Union oder andere zwischenstaatliche Einrichtungen erlaubt eine Verlagerung von politischer Herrschaft auf internationale Organisationen. Die Ermächtigung, supranationale Zuständigkeiten auszuüben, stammt allerdings von den Mitgliedstaten einer solchen Einrichtung. Sie bleiben deshalb dauerhaft die Herren der Verträge“ (Rn. 231, Hervorhebung d. Verf.).

Diese Sicht erlaubt auch den „Austritt [eines Mitgliedstaates] aus einem auf dem Prinzip der umkehrbaren Selbstbindung beruhenden Staatenverbund“ (Rn. 233). Das Urteil grenzt dieses Modell deutlich von dem eines Bundesstaates ab, in dem die Bundesrepublik nur zu einem „Gliedstaat“" würde (vgl. Rn. 179 und s. o.).

Ein wesentlicher Teil der Modellbeschreibung liegt in der Bestimmung von „,domaines resérvées “ bzw. „,red lines “, die bei einer „Vertragsunion souveräner Staaten“ und mit Blick auf die „unübertragbare und insoweit integrationsfeste Identität der Verfassung (Art. 79 Abs. 3 GG)“ (Rn. 235) nicht zu Disposition stehen.

Diese Grenzziehung von Zuständigkeiten mag der Sorge vor einer schleichenden, unmerklichen, stillschweigenden Vergemeinschaftung geschuldet sein. ${ }^{31}$ Die Verfassungsrichter zielen wahrscheinlich auf Prozesse, die als spill over ${ }^{32}$ bzw. als Wirken einer „Sachlogik“33 beschrieben und erklärt wurden. Entsprechend mahnen sie: „Das Vertrauen in die konstruktive Kraft des Integrationsmechanismus kann allerdings von Verfassungswege nicht unbegrenzt sein“ (Rn.

31 Vgl. zu ,,creeping competence“ Dougan, M., a. a. O., 655.

32 Vgl. Niemann A./Schmitter, P. C.: Neofunctionalism, in: Wiener, A./Diez, T. (Hg.): European Integration Theory, Oxford, 2009, 45-66.

33 Hallstein, W.: Die Europäische Gemeinschaft, Düsseldorf/Wien, 1979, 22. 
238). Wohl unbewusst greifen sie eine Prozesslogik des historischen Institutionalismus und der Fusionsthese auf: ${ }^{34}$ Wenn „die Organe Zuständigkeit neu begründen, erweiternd abrunden und sachlich ausdehnen dürfen, $[\ldots]$ bewegen sie sich auf einem $P f a d$, an dessen Ende die Verfügungsgewalt über ihre vertraglichen Grundlagen steht, das heißt die Kompetenz, über ihre Kompetenzen zu disponieren“ (Rn. 238, Hervorhebung d. Verf.).

Der „Staatenverbund“ ist eine Begriffsschöpfung des Verfassungsgerichts, die seit dem Maastrichter Urteil in europäischen Debatten nur begrenzt Eingang gefunden hat. Wie in derartigen Fällen zu erwarten wird der Begriff kontrovers diskutiert. Kirchhof, der „Vater“ des Konzepts im Maastricht-Urteil des Verfassungsgerichts, charakterisiert diese Kategorie als einen wichtigen Beitrag:

„Die europäische Integration führt nicht in die Alternative von Staatenbund oder Bundesstaat. Sie ist anspruchsvoller und entwickelt eine neuartige Form gesteigerter Verbundenheit selbständig bleibender Staaten - den Staatenverbund mit einer eigenen, diesem Verbund entsprechenden demokratischen Legitimation durch Rat und Europäisches Parlament. ${ }^{\text {(35 }}$

In einem ähnlichen Gedankengang wird der Begriff als ,die konstruktive Antwort des Bundesverfassungsgerichts auf die wenig hilfreichen Kennzeichnungen der Union als ,monstrum simile“"36 verstanden. Das Urteil bietet demnach , $a$ reconstructed modern concept of sovereignty “37; es überwindet klassische Vorstellungen von Souveränität nach dem Westfälischen Modell ${ }^{38}$. Das Urteil selbst argumentiert in ähnlicher Richtung: „Das Grundgesetz löst sich von einer selbstgenügsamen und selbstherrlichen souveränen Staatlichkeit“" (Rn. 223); es

„schreibt demgegenüber die Friedenswahrung und die Überwindung des zerstörerischen europäischen Staatenantagonismus als überragende politische Ziele der Bundesrepublik fest. Souveräne Staatlichkeit steht danach für einen befriedeten Raum und die darin gewährleistete Ordnung auf der Grundlage individueller Freiheit und

34 Vgl. Pierson, P.: The Path to European Integration: A Historical Institutionalist Analysis, in: Comparative Political Studies, 29/2 (1996), 123-163.

35 Kirchhof, P.: Faszination Europa, in: Frankfurter Allgemeine Zeitung v. 19. 09. 2009, 8.

36 Schröder, M.: Das Bundesverfassungsgericht als Hüter des Staates im Prozess der europäischen Integration - Bemerkungen zum Maastricht Urteil, in: Deutsches Verwaltungsblatt, 109 (1994), 320.

37 Schorkopf, F.: The European Union as an Association of Sovereign States: Karlsruhe's Ruling on the Treaty of Lisbon, in: German Law Journal 10/8 (2009), 1219-1240, hier 1224; siehe auch ders.: Der Mensch im Mittelpunkt, in: Frankfurter Allgemeine Zeitung v. 16. 07. 2009, 6.

38 Vgl. zur Definition u.a. Duchhardt, H.: „Westphalian System“. Zur Problematik einer Denkfigur, in: Historische Zeitschrift, 269/2 (1999), 305-315; Zimmer, M.: Das Westfälische System: Mythos oder Wirklichkeit?, Wiesbaden, 2008. 
kollektiver Selbstbestimmung. Der Staat ist weder Mythos noch Selbstzweck, sondern die historisch gewachsene, global anerkannte Organisationsform einer handlungsfähigen politischen Gemeinschaft“ (Rn. 224).

Andere Kommentatoren empfahlen bereits nach dem Maastricht-Urteil, den Begriff Staatenverbund als eine ,unpassende, weil ökonomisch technisch , besetzte“ Terminologie“"39 möglichst bald zu eliminieren. Als fragwürdig wird der Schlüsselbegriff Staatenverbund auch deshalb verstanden, weil ,er wegen der einseitigen Betonung der Rolle der Mitgliedstaaten nur die halbe Wahrheit wieder[gibt]. [...] Die Gemeinschaft ist nämlich nicht nur ein Verbund der Staaten, sondern auch der Bürger und der Unternehmen. “40

Noch grundsätzlicher wird der Bruch gegenüber den Vorgaben des Grundgesetzes und deren bisherigen Interpretation gesehen:

„Es gibt sprachlich und rechtlich eine ununterbrochene Kontinuität zwischen dem 1949 formulierten und noch heute in der Präambel genutzten Begriff des Vereinten Europas. Die tatsächliche Entwicklung spricht dafür, dass das Grundgesetz alle Integrationsschritte bis hin zu den Vereinigten Staaten von Europa erlaubt und zulässt. “41

Mit der Begründung eines (National-)Staates ,über alles“ versuche das Gericht, „die Möglichkeit einer Demokratisierung auf europäischer Ebene zu untergraben [...] und implizit der Gemeinschaft ein Ziel unterzuschieben, das den Gründungszwecken der europäischen Integration fremd ist ${ }^{\text {"42 }}$. Eine wesentliche Richtung der Kritik am Karlsruher Urteil sieht den Begriff und die ihm zugrunde liegende Argumentation als ein ,politisches Manifest“, das auf einer „eigenen Philosophie“ zur europäischen Integration beruht. ${ }^{43}$

\section{Transformation des Staates: Ausbau des Fusionspfades?}

Gegenüber den Erwartungen, die aus Konzepten des Staatenverbundes und der Union als „Rettung des Nationalstaates“ abgeleitet werden, analysiert die Fusi-

39 Ipsen, H. P.: Zehn Glossen zu Maastricht-Urteil, in: Europarecht, 29/1 (1994), 21.

40 Vgl. Everling, U.: Das Maastricht-Urteil des Bundesverfassungsgerichts und seine Bedeutung für die Entwicklung der Europäischen Union, in: Integration, 17/3 (1994), 165-175, hier 167.

41 Lenz, C.: Ausbrechender Rechtsakt, in: Frankfurter Allgemeine Zeitung vom 08. 08. 2009, 7.

42 Weiler, J.: Der Staat ,über alles‘. Demos, Telos und die Maastricht-Entscheidung des Bundesverfassungsgerichts, in: Jean Monnet Working Papers, 7 (1995), 3.

43 Tomuschat, C.: The Ruling of the German Constitutional Court on the Treaty of Lisbon, in: German Law Journal, 10/8 (2009), 1259-1262, hier 1259. 
onsthese: Bei dem Austarieren des magischen Dreiecks bleiben die Mitgliedstaaten zwar in Grundfragen der Systemgestaltung „Herren der Verträge“ (Rn. 231), aber infolge konkreter Schritte der Politikgestaltung verändern sie selbst bewusst oder unbewusst wesentliche Charakteristika staatlichen Handelns. Zur Problembewältigung (der „Rettung“) übertragen die Mitgliedstaaten in einer Mehrebenenverfassung ${ }^{44}$ zentrale staatliche Zuständigkeiten und wirken dann über ihre Repräsentanten an der Ausübung dieser Kompetenzen mit.

Die Spannung zwischen den Fundamentalzielen spiegelt sich - nach dieser Sicht - insbesondere in der Arbeit des Europäischen Rates wider. Ausgangspunkt ist eine Analyse, die von den skizzierten Spannungen in dem magischen Dreieck (siehe oben) ausgeht. Danach befinden sich die Staats- und Regierungschefs in einem (Ebenen-)Dilemma, zwischen dem Bestreben nach einem optimalen Politikgestaltungsraum, dem Problembewältigungsinstinkt, und der Absicherung der historisch gewachsenen Zuständigkeiten des Mitgliedstaates, dem Souveränitätsreflex, abwägen zu müssen. Die Suche nach einem Gleichgewicht führt zu einer Kluft zwischen den eigenen hohen Erwartungen an die Problembewältigungsfähigkeit des EU-Systems und den ihm zur Verfügung gestellten begrenzten Zuständigkeiten und Ressourcen (capability-expectations gap $^{45}$ ), die zwar bei jedem politischen System zu beobachten ist, aber in der EU spezifische und besonders intensive Formen annimmt.

Das mit dem „Ebenendilemma“ verknüpfte „Entscheidungsdilemma“ besteht darin, dass die Mitgliedstaaten für eine effiziente und effektive Problemlösung bei der Vorbereitung, Herstellung, Durchführung und Kontrolle verbindlicher Entscheidungen auf nationalstaatliche Handlungsautonomie - insbesondere in Form der Aufgabe des Vetos im Rat - verzichten müssten.

Um das doppelte Dilemma zu überwinden, haben die Staats- und Regierungschefs im Rahmen von Vertragsänderungen in der Vergangenheit und erneut im Lissabonner Vertrag sowohl die Handlungsfähigkeit der Europäischen Union ausgebaut als auch parallel ihren nationalstaatlichen Einfluss gestärkt. Dabei verlagerten sie in komplexen Verhandlungspaketen zunehmend staatliche $\mathrm{Zu}$ ständigkeiten auf die EU-Ebene und stärkten supranationale Institutionen und Verfahren. Gleichzeitig weiteten nationale Regierungen und Verwaltungen ihre

44 Vgl. zum Begriff Pernice, I.: Das Verhältnis europäischer zu nationalen Gerichten im europäischen Verfassungsbund, Berlin, 2006.

45 Vgl. Hill, C.: The Capability-Expectations Gap, or Conceptualizing Europe's International Role, in: Journal of Common Market Studies, $31 / 3$ (1993), 305-328. 
eigenen Beteiligungsmöglichkeiten bei der Politikgestaltung des EU-Systems extensiv aus.

In der hier vertretenen These bildet der fusionierte EU-Mehrebenenstaat - nach den Stufen des Territorial-, National-, Verfassungs- und Wohlfahrtstaates - eine weitere Phase in der historischen Entwicklung europäischer Staaten. Der Vertrag von Lissabon dokumentiert demnach eine nächste Stufe in einer Langzeitbetrachtung staatlicher Funktionen und Strukturen.

In der Fortschreibung des Fusionstrends haben sich die Mitgliedstaaten in mehreren Abschnitten des Lissabonner Vertragswerks für die Politikgestaltung des EUSystems ehrgeizige Ziele gesetzt und eine umfassende Liste an gemeinsamen Aufgaben vorgenommen. Die Präambel (Spiegelstriche 8 bis 12) und Artikel 3 des EUV führen einen Katalog von Zielen auf, die wesentliche Bereiche staatlichen Handelns umfassen. In Art. 2 bis 6 AEUV haben die „Herren der Verträge“ die Bereiche der Zuständigkeiten konkret aufgelistet. Im Vergleich zu dem Vertrag von Nizza haben sie noch weitere Bereiche hinzugefügt oder expliziter formuliert. Weitere Ziele und Aufgabendefinitionen haben die Vertragsschöpfer zu einzelnen Bereichen der Unionspolitiken formuliert; so machen sie in Art. 21 EUV weitere und konkretere Vorgaben zum „Auswärtigen Handeln der Union“.

Addiert man die im Vertrag angesprochenen Politikbereiche auf, so haben die Vertragsschöpfer der Union eine Agenda an Aufgaben aufgetragen, die weitgehend denen der Mitgliedstaaten entsprechen. Sie entwerfen die Union als ein umfassendes - fast , catch all “- System, in welchem sie Probleme auf der nationalen Tagesordnung auch durch die EU-Institutionen angehen wollen. ${ }^{46}$

Bei dieser - zugegebenermaßen thesenhaften - Charakterisierung einer staatsähnlichen Agenda ist zu betonen, dass die Verträge die Art der Zuständigkeit der EU und die Verfahren ihrer Ausübung erheblich variieren und dabei Vorbehalte gegen einen zu starken Kompetenztransfer in mehreren Ausprägungen einbringen.

Von besonderem Interesse für die Fusionsthese ist die Liste der Bereiche, die als „geteilte Zuständigkeit“ (Art. 2 Abs. 2 AEUV) ausgeschildert sind und damit auch eine ,geteilte Souveränität“47 signalisieren. Nach den Bestimmungen zu dieser Art von Zuständigkeit verlieren die Mitgliedstaaten ihre Zuständigkeit,

46 Vgl. mit einem anderen Tenor BVerfG, a. a. O., Rn. 351.

47 Vgl. Urteil des Tschechischen Verfassungsgerichts zum Vertrag von Lissabon vom 03.11. 2009, online unter http://www.concourt.cz/clanek/GetFile?id=2150 (tschechische Version). 
wenn und insofern die Union diese Kompetenzen in den aufgelisteten Politikbereichen nutzt. Bei dieser Kategorie schreiben die Vertragsschöpfer der Union durchgängig ein Verfahren vor, das - zur Steigerung der Effizienz - supranational ausgerichtet ist: Die Unionsorgane entscheiden nach dem ,ordentlichen Gesetzgebungsverfahren“, also auf Grund des Vorschlagmonopols der Kommission, in Mitentscheidung zwischen Rat und Europäischem Parlament (EP), einschließlich der Möglichkeit, im Rat mit qualifizierter Mehrheit abzustimmen, und im Schatten einer möglichen Prüfung durch den Gerichtshof der Europäischen Union (EuGH). Mit diesem Verfahren gibt jeder einzelne Mitgliedstaat auch sein Letztentscheidungsrecht auf, mithilfe eines Vetos die Politikgestaltung durch die EU-Institutionen blockieren zu können.

Im Vergleich zu den vorangegangenen Verträgen haben die Mitgliedstaaten die Anwendungsbereiche dieses Verfahrens bei der Ausübung der Zuständigkeiten wesentlich erweitert. ${ }^{48}$ Gleichzeitig haben sie für diese Art von Zuständigkeiten die Rolle der nationalen Parlamente durch das Subsidiaritätsverfahren gestärkt und damit Beteiligungsmöglichkeiten von unterschiedlichen Akteursgruppen auf mehreren Ebenen staatlichen Handelns verflochten.

Dem Problembewältigungsinstinkt stehen in demselben Dokument mehrere und neuere Ausprägungen des Souveränitätsreflexes gegenüber, die bis auf wenige Ausnahmen - so der Möglichkeit des Austritts - jedoch keinen Rückbau in Richtung Nationalstaat signalisieren, sondern den Zuwachs an Politikgestaltungsmöglichkeiten begrenzen und kontrollieren wollen. So sehen die „Herren der Verträge" vor, dass der Rat bei einigen als besonders sensitiv eingestufte Bereiche der Gesetzgebung sowie bei der Festlegung von Eigenmitteln (Art. 311 AEUV) und des mehrjährigen Finanzrahmens (Art. 312 AEUV) weiterhin nach den Regeln der „besonderen Gesetzgebungsverfahren“ einstimmig beschließt.

Einige Vorschriften, so insbesondere zur Rückübertragung von geteilten Zuständigkeiten, nachdem die Union ihre Nutzung beendet (vgl. Art. 2 Abs. 2 AEUV), formulieren nur gültiges Recht. Die Erwartung zunehmender Fusion ist so nicht unmittelbar falsifiziert, sondern modifiziert.

Mit Blick auf zukünftige Wirkungen des Lissabonner Vertrags erwartet diese These bei einem Durchspielen der geschriebenen Vorgaben des Vertragsrechts eine Praxis, die - in Verstärkung jahrzehntelanger Trends - durch ausgeprägte

48 Vgl. Hofmann, A./Wessels, W.: Der Vertrag von Lissabon - eine tragfähige und abschließende Antwort auf konstitutionelle Grundfragen?, in: Integration 31/1 (2008), 3-20. 
Tendenzen zur Fusion in der institutionellen Architektur des EU-Mehrebenensystems geprägt sein wird. Entgegen Forderungen - auch des Verfassungsgerichts - nach „Bildung eines durchsetzungsfähigen Mehrheitswillens“ und nach „greifbaren Verantwortungszusammenhängen“ (Rn. 247) werden weitere „VetoSpieler “49 - so insbesondere nationale Parlamentskammern und nicht zuletzt das Karlsruher Gericht selbst - zusätzliche Verfahren, welche der Lissabonner Vertrag - und im deutschen Fall auch das Verfassungsgericht - anbietet bzw. fordert, intensiv nutzen. Diese umfassende Beteiligung wird paradoxerweise zu einem höheren Grad an Komplexität des Gesamtsystems führen. Ein Gewinn an Transparenz in einzelnen Phasen und Stationen des Entscheidungsprozesses reduziert in der Summe die Eindeutigkeit bei der angemahnten „Integrationsverantwortung“ (Rn. 238-245 u. a., Leitsatz 2 und Begleitgesetz ${ }^{50}$ ). Im Lichte einer Typologie von Formen demokratischer Systeme ist zu erwarten, dass die angemahnten Anpassungen in der institutionellen Architektur den bereits vorhande-

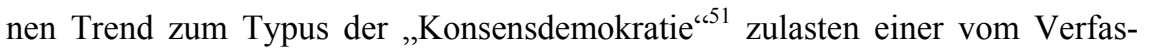
sungsgericht beschriebenen „Mehrheitsdemokratie“ ${ }^{\text {“52 }}$ fördern.

Sieht man derartige Entwicklungen im Lissabonner Vertrag angelegt, so ergibt sich ein offensichtliches „Rätsel“: Wie ist zu erklären, dass die entscheidenden vertragsverändernden Konferenzen nationaler Regierungen und entsprechende Ratifizierungsprozesse nationaler Verfassungsorgane erneut Handlungsmöglichkeiten der EU zur Problembewältigung zulasten nationaler Autonomieansprüche und Letztentscheidungsrechte ausgebaut haben? Der Einstieg zur Beantwortung beginnt mit der Wahl der handelnden Subjekte. Bewusst wurden hier Formulierungen genutzt, welche die „Hohen Vertragsparteien“, die „Vertragsschöpfer“ oder mit den Begriffen des Bundesverfassungsgericht die „Herren der Verträge“ als Gestalter des EU-Systems identifizieren: Die Ausweitung der gemeinsam angegangenen Politikbereiche mit der entsprechenden Zunahme an Aufgaben des EU-Systems, wie die Mitgliedstaaten sie seit den Gipfeln von Den Haag 1969 und Paris 1972 verfolgen, ist nicht durch eine Usurpation ${ }^{53}$ seitens supranationa-

49 Vgl. zum Begriff Tsebelis, G.: Veto Players. How Political Institutions Work, New York, 2002; Kaiser, A.: Tsebelis, Veto Players, in: Kailitz, S. (Hg.): Schlüsselwerke der Politikwissenschaft, Wiesbaden, 2007, 464-468.

50 Integrationsverantwortungsgesetz, BGB1 I, 2009, 3022-3035.

51 Vgl. Lijphart, A.: Patterns of Democracy, New Haven, 1999; Scharpf, F. W.: Versuch über Demokratie in Verhandlungssystemen, MPIfG Discussion Paper, 92/9, Köln, 1992.

52 Vgl. zur Typologie Lijphart, A., a. a. O., 42-47.

53 Vgl. zum Begriff Kerber, M.: Plädoyer zur Verfassungsbeschwerde zum Lissabon-Vertrag vom 10./11.02. 2009, online unter http://www.europolis-online.org/index.php?id=20. 
ler EG-Organe verursacht worden, sondern folgte und folgt den jeweiligen Konstellationen nationaler Präferenzen der Mitgliedstaaten, wie sie durch die staatlichen Exekutiven in die jeweiligen Regierungskonferenzen eingebracht und verhandelt wurden. ${ }^{54}$ Weder das $\mathrm{EP}$, dessen demokratische Legitimität vom Verfassungsgericht als „zusätzliche Quelle“ (Rn. 271), das heißt als „sekundär“ gegenüber nationalen Parlamenten eingestuft wird, noch die Kommission, die der Amtsanmaßung beschuldigt wird, ${ }^{55}$ noch der EuGH, dessen Rechtsprechung wieder immer als ,, ultra vires “, als ausbrechender Rechtsakt, bezeichnet wird, ${ }^{56}$ haben diese Vertragsänderung beschlossen und dann nationalen Parlamenten und Referenden aufgezwungen - auch wenn diese Organe in den vorbereitenden Debatten auf einzelne Sektoren bzw. in spezifischen Regelwerken jeweils begrenzte prä-konstitutionelle Einflüsse ausgeübt haben mögen. Die Schlüsselrolle bei der beschriebenen Systemgestaltung zur Verbesserung der Problembewältigung spielt vielmehr das Gremium der höchsten politischen Entscheidungsträger der Mitgliedstaaten: Der Europäische Rat der Staats- und Regierungschefs hat als konstitutioneller Architekt ${ }^{57}$ alle entscheidenden Beschlüsse vorbereitet und in der häufig so charakterisierten „Nacht der langen Messer“ in einem der üblichen Verhandlungspakete beschlossen. Auch den Lissabonner Vertrag hat der Europäische Rat weitgehend am Ende der deutschen Präsidentschaft 2007 entschieden. ${ }^{58}$ Die Verstärkung des Fusionspfades durch den Vertrag von Lissabon ist demnach als Reaktion der europäischen Staaten auf grundsätzliche Veränderungen des Wirkungskontextes zu verstehen.

\section{Vom Vertragstext zur Vertragspraxis: zum Realitätstest der Interpretationsangebote}

Mit Bezug auf die Kontroversen um mögliche Antworten auf die Schlüsselfrage erlauben das Lissabonner Dokument und dessen Interpretationen - insbesondere

54 Vgl. generell Moravscik, A.: The Choice for Europe: Social Purpose and State Power from Messina to Maastricht, Ithaca, NY, 1998; Christiansen, T./Reh, C.: Constitutionalising the European Union, London, 2009.

55 Vgl. u. a. Kerber, M., a. a. O.; Vaubel, R.: The European Institutions as an Interest Group, London, 2009.

56 Vgl. u.a. Behrens, P.: Aufruf: Stoppt das Bundesverfassungsgericht, in: Frankfurter Allgemeine Zeitung (Briefe an die Herausgeber) v. 14.09. 2009, 20.

57 Vgl. Wessels, W.: Das politische System der Europäischen Union, Wiesbaden, 2008, 37-39.

58 Vgl. Weidenfeld, W:: Die Bilanz der Europäischen Integration 2007, in: Jahrbuch der Europäischen Integration 2007, Baden-Baden, 2008, 13-24; Wessels, W./Faber, A.: Vom Verfassungskonvent zurück zur „Methode Monnet“? Die Entstehung der „Road map“ zum EU-Reformvertrag unter deutscher Ratspräsidentschaft, in: Integration, 30/4 (2007), 370-381. 
durch das Urteil des Bundesverfassungsgerichts - einen nächsten und vertiefenden Schritt zur Analyse des politischen Systems im Werden und dessen mögliche Auswirkungen auf den gewachsenen Nationalstaat. Die konzeptionelle Unsicherheit, die sich in den Argumenten zu den skizzierten Modellen niederschlagen, kann der Text des Lissabonner Vertrags und die damit verbundenen Charakterisierungen und Auslegungen jedoch nicht abschließend überwinden. Unsere Grundsatzdebatte wird jedoch nachhaltig durch zusätzliche Elemente angereichert.

Angesichts der Bemühungen der Vertragsschöpfer, im Vertragstext die drei Grundziele erneut und anders auszutarieren, ist zunächst eine erste Zwischenbilanz im Hinblick auf die skizzierten Modelle angebracht.

Die beiden Endpunkte - übliche internationale Organisation hier und klassischer Bundesstaat dort - greifen nicht als Gesamtcharakterisierungen; sie sind aber nicht einfach als utopische Referenz- und theoretische Eckpunkte der Debatte abzutun. In einzelnen Vertragsartikeln sind Entwicklungen in beide Richtungen angelegt.

Für eine Gesamtsicht ertragreicher ist jedoch eine Diskussion der Zwischen- und Mischformen. Auch zu diesen Formen sind in jeweils unterschiedlicher Dichte und mit Variationen Spuren bzw. Belege im Vertragstext und dessen Auslegungen zu finden.

Ein Resultat des gleichzeitigen Verfolgens aller drei Eckpunkte des magischen Dreiecks ist eine noch zunehmende Komplexität. Nicht ein „Entweder-oder“, sondern ein „Sowohl-als-auch“ ist vorläufig aus den Texten herauszulesen. Das Urteil des Bundesverfassungsgerichts orientiert sich zwar im Tenor auf das Modell des Staatenverbundes, aber in einigen Konsequenzen stärkt es unwillentlich den Prozess der Fusion staatlichen Handelns im Mehrebenensystem.

Für eine abgesicherte Abwägung des Befundes mit Blick auf Realitätsnähe und die normativen Implikationen ist jedoch der Zeitpunkt noch nicht gekommen. Angesichts derartiger Kontroversen zählt es zu den Floskeln wissenschaftlicher Untersuchungen, auf die Ungewissheit zukünftiger Entwicklungen zu verweisen. Aus der hier zur Diskussion gestellten Sicht lässt sich ein Satz von Erwartungen zur weiteren Beobachtung der im Dezember 2009 begonnenen Vertragspraxis ableiten. Zentral ist dafür folgende These: Die Mitgliedstaaten werden, insbesondere im und durch den Europäischen Rat auf der gerade für diese Institution ausgebauten Basis - er hat sich selbst 150mal im Lissabonner Vertrag verankert-, auch weiterhin und verstärkt im EU-System Lösungen für jegliche Art 
von Herausforderungen suchen und dabei den Souveränitätsreflex implizit beachten.

Geht man von einer derartigen vitalen Bedeutung der Union für die Mitgliedstaten aus, so ist auch zu erwarten, dass der Vertragstext noch nicht die Endstufe der Entwicklung der Union und damit der Rolle des EU-Systems für die Evolution der europäischen Staaten darstellt. Auch wenn die Staats- und Regierungschefs „,in absehbarer Zukunft keine weiteren Änderungen“ anvisieren, ${ }^{59}$ so ist ein erneuter Druck zur Vertragsrevision nicht auszuschließen: Die Vorgabe der Hohen Vertragsparteien, diesen Vertrag als „eine neue [aber eben nicht endgültige, d. Verf.] Stufe bei der Verwirklichung einer immer engeren Union der Völker Europas“ (Art. 1 EUV) einzuordnen, bietet die Möglichkeit zu weiteren Vertragsänderungen, für die das Urteil des Verfassungsgerichts zunächst deutliche Schranken errichtet.

Die Diskussionen um die Beziehungen der EU und der (National-)Staaten reflektieren Trends im Zeitgeist. Vielleicht markiert diese quasi-konstitutionelle Grundsatzdebatte eine Wegmarke, die sich nicht nur in der realen Europapolitik der nächsten Phase europäischer Geschichte bemerkbar machen, sondern zu einer auch wissenschaftlichen Wende führen kann; demnach könnten traditionelle Denkschulen konventionelle Argumentationslinien auf der Suche nach Antworten auf die Schlüsselfrage erneut aufgreifen und weiterentwickeln, wobei die Interpretationen des Vertrags und des Urteils des deutschen und anderer Verfassungsgerichte abweichende Richtungen nehmen können. ${ }^{60}$

Freilich kann das Inkrafttreten des Vertrags auch eine Epoche dieser Auseinandersetzung mehrerer Denkschulen und Strategien zunächst abschließen. Das Tagesgeschäft in einer veränderten Architektur steht dann im Vordergrund der politischen und wissenschaftlichen Aufmerksamkeit; die Vertragspraxis mag dann exemplarische Antworten zur Schlüsselfrage geben, ohne sie jeweils umfassend zu thematisieren. Die konzeptionelle Erbschaft, der acquis des Schulstreits dieses Jahrzehnts, kann dann fortwirken und bei der Anwendung des Lissabonner Vertrags jeweils begrenzt aktualisiert werden, ohne jedoch unmittelbar in eine politisch relevante Grundsatzkontroverse zu münden.

59 Schlussfolgerung des Europäischen Rates vom 14.12. 2007, online unter: http://www.consilium.europa. eu/uedocs/cms_data/docs/pressdata/de/ec/97683.pdf.

$60 \mathrm{Vgl}$. Hofmann, A./Slosarcik, I.: National Sovereignty and European Integration - Mutually Exclusive? The Treaty of Lisbon and a View from the Courts, EUPOLIS Working Paper, 1, Köln, 2009. 